\title{
A study of steady-state heat conduction for longitudinal fin of rectangular profile
}

\author{
ZUO Haoyu \\ North China Electric Power University (Baoding) \\ Baoding, China \\ zuohaoyu1994@163.com
}

\begin{abstract}
Through the appropriate assumptions, the mathematical model was derived in longitudinal fin of rectangular profile, use the finite difference method for the numerical solution to the model, then use the fluent software to draw a two-dimensional temperature distribution within the region. The results show good agreement with different solving methods, and the influence of different parameters for the twodimensional temperature distribution is analyzed.
\end{abstract}

Keywords- longitudinal fin of rectangular profile; numerical solution; heat conduction

\section{INTRODUCTION}

A rectangular fin, the height of the fin is $H$, the thickness is $\delta$, long in length direction is $l$, the temperature of the fin root is $t_{0}$, the fluid temperature near the fin is $t_{\infty}$, the surface heat transfer coefficient is $h$. Assuming that $t_{0}>$ $t_{\infty}$, And $h$ is constant, The area along the height direction is $A_{\mathrm{c}}$, the perimeter of the cross section is $P, P / A_{\mathrm{c}} \approx 2 / \delta$.

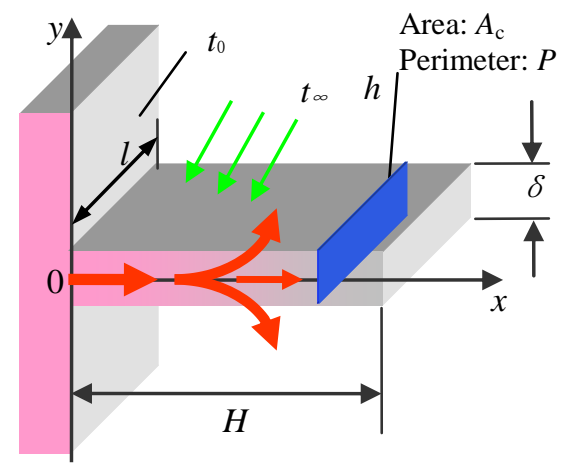

Figure 1. the schematic diagram of longitudinal fin of rectangular profile

\section{THE ANALYSIS OF THE RESEARCH OBJECT AND ITS MATHEMATICAL MODEL}

\section{A. The analysis and build of the model}

Ignore the temperature difference in the thickness direction, the fins temperature only changes in the height direction, only study the average temperature changing in the height direction of each cross section. Use the first boundary condition on the top of the fin, the surface of the fin is having convective heat transfer with the fluid nearby, but in reality, the heat transfer through the top of fin is very small, so conduct the adiabatic process. The heat transfer through the upper surface and lower surface can't be ignored, so we introduce the virtual heat source.

Differential equations of this heat conduction problem:

$$
\frac{d^{2} t}{d x^{2}}+\dot{\Phi}=0
$$

According to the above assumptions, the boundary conditions are:

$$
\begin{gathered}
x=0, t=t_{0} \\
x=H, \frac{d t}{d x}=0
\end{gathered}
$$

Among them:

$$
\dot{\Phi}=-\frac{h P\left(t-t_{\infty}\right)}{A_{C}}
$$

\section{B. Analysis and solution}

Introduce the over temperature $\theta=t-t_{\infty}$, and $m^{2}=h P / \lambda A_{c}$, thus makes the equation homogeneous and easy to solve.

The equation now is:

$$
\frac{d^{2} \theta}{d x^{2}}-m^{2} \theta=0
$$

Boundary conditions are:

$$
x=0, \theta=\theta_{0}
$$




$$
x=H, \frac{d \theta}{d x}=0
$$

The solution are:

$$
\theta=\theta_{0} \frac{\operatorname{ch}[m H(1-x / H)]}{\operatorname{ch}(m H)}
$$

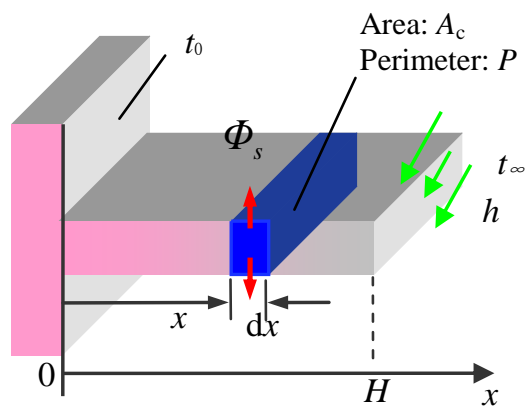

Figure 2. the schematic diagram of the virtual heat source.

\section{The basic process of numerical solution}

1) The basic process of one-dimensional numerical solution

Conduct the one-dimensional numerical analysis of temperature field within the area of the fin (do adiabatic boundary treatment), the solution process is as follows:

Discrete the region: to discrete the rectangular area, the region in the height direction is divided into $N$ sub-region, and $N+1$ nodes, the step size is $\Delta x=H / N$, from left to right are the nodes $0-N$.

Establish discrete node equations.

According to energy conservation relations, the discrete equation of the internal node $N$ is:

$$
\lambda A_{C} \frac{t_{n-1}-t_{n}}{\Delta x}+\lambda A_{C} \frac{t_{n+1}-t_{n}}{\Delta x}+h P \Delta x\left(t_{\infty}-t_{n}\right)=0
$$

Left boundary nodes:

$$
t_{0}=t_{0}
$$

Right boundary nodes:

$$
\lambda A_{C} \frac{t_{N-1}-t_{N}}{\Delta x}+h P \frac{1}{2} \Delta x\left(t_{N-1}-t_{N}\right)=0
$$

2) The basic process of two-dimensional numerical solution

Conduct the two-dimensional numerical analysis of temperature field within the area of the fin(do the third type of boundary conditions treatment), the solution process is as follows.

Figure 3 is the discretion of the rectangular area.

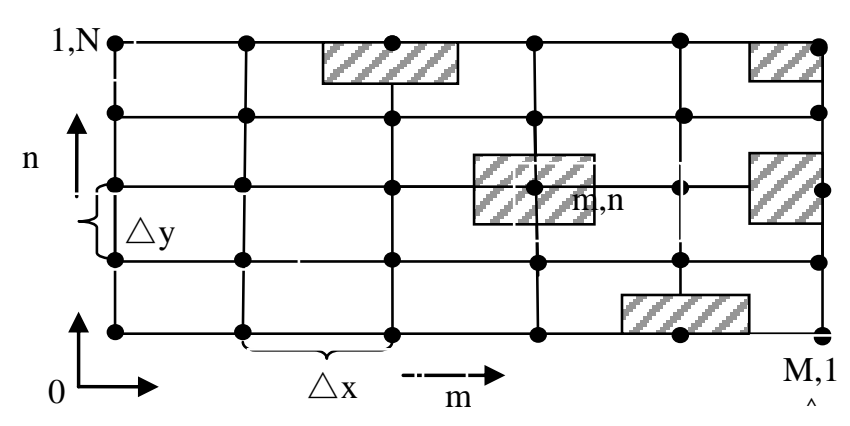

Figure 3. the discretion of the rectangular area

According to the energy conservation relation, to give temperature expression of the internal node $(m, n)$ :

$$
t_{m, n}=\frac{\left(\frac{\Delta y}{\Delta x} t_{m-1, n}+\frac{\Delta y}{\Delta x} t_{m+1, n}+\frac{\Delta x}{\Delta y} t_{m, n-1}+\frac{\Delta x}{\Delta y} t_{m, n+1}\right)}{2 \frac{\Delta x}{\Delta y}+2 \frac{\Delta y}{\Delta x}}
$$

Before you begin to format your paper, first write and save the content as a separate text file. Keep your text and graphic files separate until after the text has been formatted and styled. Do not use hard tabs, and limit use of hard returns to only one return at the end of a paragraph. Do not add any kind of pagination anywhere in the paper. Do not number text heads-the template will do that for you.

The upper boundary nodes (not including the left and right nodes):

$$
t_{m, N}=\frac{\lambda\left(\frac{\Delta x}{2 \Delta y} t_{m-1, N}+\frac{\Delta y}{2 \Delta x} t_{m+1, N}+t_{m, N-1}\right)+h \Delta x t_{\infty}}{\lambda\left(\frac{\Delta x}{\Delta y}+\frac{\Delta y}{\Delta x}\right)+h \Delta x}
$$

The lower boundary nodes (not including left and right nodes):

$$
t_{m, N}=\frac{\lambda\left(\frac{\Delta y}{2 \Delta x} t_{m-1,1}+\frac{\Delta y}{2 \Delta x} t_{m+1,1}+\frac{\Delta x}{\Delta y} t_{m, 2}\right)+h \Delta x t_{\infty}}{\lambda\left(\frac{\Delta x}{\Delta y}+\frac{\Delta y}{\Delta x}\right)+h \Delta x}
$$

The node in the upper right corner:

$$
t_{m, N}=\frac{\lambda\left(\frac{\Delta y}{\Delta x} t_{M-1, N}+\frac{\Delta x}{\Delta y} t_{M, N-1}+\right)+h(\Delta x+\Delta y) t_{\infty}}{\lambda\left(\frac{\Delta x}{\Delta y}+\frac{\Delta y}{\Delta x}\right)+h(\Delta x+\Delta y)}
$$

The node in the upper right corner: 


$$
t_{m, N}=\frac{\lambda\left(\frac{\Delta y}{\Delta x} t_{M-1,1}+\frac{\Delta x}{\Delta y} t_{M, 2}+\right)+h(\Delta x+\Delta y) t_{\infty}}{\lambda\left(\frac{\Delta x}{\Delta y}+\frac{\Delta y}{\Delta x}\right)+h(\Delta x+\Delta y)}
$$

\section{CACULATION}

The first set of conditions contain such given main parameters: the thermal conductivity $\lambda=100 \mathrm{~W} / \mathrm{m} / \mathrm{K}$, the surface heat transfer coefficient $h=50 \mathrm{~W} / \mathrm{m}^{2} / K$, the thickness $\delta=0.02 \mathrm{~m}$, the height $H=0.12 \mathrm{~m}$, the temperature of fin root $t_{0}=100^{\circ} \mathrm{C}$, the fluid temperature near the fin $t_{f}=20^{\circ} \mathrm{C}$

To study the two-dimensional temperature field distribution under different parameters, give a second set of conditions, the thermal conductivity $\lambda=10 \mathrm{~W} / \mathrm{m} / \mathrm{K}$, the surface heat transfer coefficient $h=400 \mathrm{~W} / \mathrm{m}^{2} / K$, the other conditions remain unchanged

The second set of conditions are only calculated and compared in chapter $\mathrm{C}$, and the rest are based on the calculation of the first set of conditions.

\section{A. Analytical solution}

Substitute the nodes and obtain the temperature distribution of the corresponding node, as shown in Table 1

Table 1. the theoretical solution of each node

\begin{tabular}{cc}
\hline $\begin{array}{c}\text { Node } \\
\text { Number }\end{array}$ & Temperature \\
\hline 0 & 100 \\
1 & 96.23 \\
2 & 92.85 \\
3 & 89.85 \\
4 & 87.19 \\
5 & 84.89 \\
6 & 82.91 \\
7 & 81.25 \\
8 & 79.91 \\
9 & 78.87 \\
10 & 78.13 \\
11 & 77.69 \\
12 & 77.54 \\
\hline
\end{tabular}

\section{B. One-dimensional numercial analysis solution}

By the analysis of numerical solution process, the area will be divided into 12 sub-regions and 13 nodes, the step size $\Delta x=H / N=0.01 \mathrm{~m}$.

When the error between two iterations is less than $10^{-6}$, the result convergence. It is shown in Table 2.
Table 2. the iteration results of each node

\begin{tabular}{|c|c|c|c|c|c|c|c|c|c|c|c|c|c|}
\hline \multirow{2}{*}{ Iterations } & \multicolumn{13}{|c|}{ er } \\
\hline & 0 & 1 & 2 & 3 & 4 & 5 & 6 & 7 & 8 & 9 & 10 & 11 & 12 \\
\hline 1 & 100 & 100 & 100 & 100 & 100 & 100 & 100 & 100 & 100 & 100 & 100 & 100 & 100 \\
\hline 200 & & 96.57 & 3.52 & 90.83 & 8.49 & 86.46 & 84.73 & 83.30 & 82.14 & 81.25 & 80.63 & 80.25 & 80.13 \\
\hline 300 & & 96. & .07 & 17 & 7.62 & 41 & 1 & 81.93 & 80.65 & 79.66 & 8.96 & 8.54 & 40 \\
\hline & & 96.23 & 92.86 & & 20 & 89 & 2 & 1.26 & 79.92 & 78.88 & 78.14 & 77 & 77.55 \\
\hline 900 & & 96.23 & 92. & 5 & 0 & 89 & 1 & 1.26 & 79.92 & 78.88 & 78.14 & 77.70 & 77.55 \\
\hline & & 96. & 92.86 & 5 & 7.20 & 84.89 & 1 & 81.26 & 79 & 8 & 78.14 & 77.70 & 77.55 \\
\hline & & 96.23 & 92 & & & 84 & & 81.26 & 75 & 78.88 & 14 & 77.70 & 77.55 \\
\hline & & 96.23 & 92 & 89 & & & & 81. & 79.91 & 78 & 8.14 & & 77.55 \\
\hline & & 96.23 & 92.86 & 89 & 8 & 84.89 & 1 & 81.26 & 79.91 & 78 & 78.14 & 77 & $\begin{array}{l}77.55 \\
\end{array}$ \\
\hline & & 96.23 & 92 & 89. & 87.20 & 84.89 & 82. & 1.26 & 9.91 & 8.88 & 8.14 & 77.70 & 7.5 \\
\hline & & & & & & & & & & & & & \\
\hline
\end{tabular}

Two-dimensional numercial analysis solution

By the analysis of numerical solution process, the area will be divided into 96 sub-regions, 13 nodes horizontal and 9 nodes in vertical direction and the step size $\Delta$ $x=H / N=0.01 \mathrm{~m}$.

When the error between two iterations is less than 10-6, the result convergence. It is shown in Table 3 .

Table 3. the two-dimensional numerical solution of each node

\begin{tabular}{c|c|c|c|c|c|c|c|c|c|c|c|c|c}
\hline \multirow{2}{*}{$\mathrm{Y}(\mathrm{m})$} & \multicolumn{10}{|c|}{$\mathrm{X}(\mathrm{m})$} \\
\cline { 2 - 13 } & 0.01 & 0.02 & 0.03 & 0.04 & 0.05 & 0.06 & 0.07 & 0.08 & 0.09 & 0.1 & 0.11 & 0.12 \\
\hline 0 & 100 & 95.98 & 92.46 & 89.30 & 86.50 & 84.02 & 81.86 & 80.02 & 78.47 & 77.21 & 76.24 & 75.55 & 75.14 \\
0.0025 & 100 & 96.06 & 92.54 & 89.38 & 86.57 & 84.09 & 81.93 & 80.08 & 78.53 & 77.27 & 76.30 & 75.61 & 75.20 \\
0.005 & 100 & 96.12 & 92.59 & 89.43 & 86.62 & 84.14 & 81.98 & 80.13 & 78.58 & 77.32 & 76.34 & 75.65 & 75.24 \\
0.0075 & 100 & 96.15 & 92.63 & 89.47 & 86.65 & 84.17 & 82.01 & 80.16 & 78.60 & 77.34 & 76.37 & 75.68 & 75.26 \\
0.01 & 100 & 96.16 & 92.64 & 89.48 & 86.66 & 84.18 & 82.02 & 80.17 & 78.61 & 77.35 & 76.38 & 75.69 & 75.27 \\
0.0125 & 100 & 96.15 & 92.63 & 89.47 & 86.65 & 84.17 & 82.01 & 80.16 & 78.60 & 77.34 & 76.37 & 75.68 & 75.26 \\
0.015 & 100 & 96.12 & 92.59 & 89.43 & 86.62 & 84.14 & 81.98 & 80.13 & 78.58 & 77.32 & 76.34 & 75.65 & 75.24 \\
0.0175 & 100 & 96.06 & 92.54 & 89.38 & 86.57 & 84.09 & 81.93 & 80.08 & 78.53 & 77.27 & 76.30 & 75.61 & 75.20 \\
0.02 & 100 & 95.98 & 92.46 & 89.30 & 86.50 & 84.02 & 81.86 & 80.02 & 78.47 & 77.21 & 76.24 & 75.55 & 75.14 \\
\hline
\end{tabular}

The temperature results under the second set of conditions are shown in table 4.

Table 4. the solution of each node under the second condition

\begin{tabular}{c|c|c|c|c|c|c|c|c|c|c|c|c|c}
\hline \multirow{2}{*}{$\mathrm{Y}(\mathrm{m})$} & \multicolumn{10}{|c|}{$\mathrm{X}(\mathrm{m})$} \\
\cline { 2 - 12 } & 0 & 0.01 & 0.02 & 0.03 & 0.04 & 0.05 & 0.06 & 0.07 & 0.08 & 0.09 & 0.1 & 0.11 & 0.12 \\
\hline 0 & 100 & 59.71 & 41.83 & 32.14 & 26.76 & 23.77 & 22.10 & 21.17 & 20.65 & 20.36 & 20.21 & 20.12 & 20.08 \\
0.0025 & 100 & 62.98 & 43.76 & 33.22 & 27.36 & 24.10 & 22.28 & 21.27 & 20.71 & 20.40 & 20.22 & 20.13 & 20.08 \\
0.005 & 100 & 65.13 & 45.15 & 34.01 & 27.80 & 24.34 & 22.42 & 21.35 & 20.75 & 20.42 & 20.24 & 20.14 & 20.09 \\
0.0075 & 100 & 66.36 & 45.98 & 34.48 & 28.07 & 24.49 & 22.50 & 21.39 & 20.78 & 20.44 & 20.25 & 20.14 & 20.09 \\
0.01 & 100 & 66.76 & 46.26 & 34.64 & 28.16 & 24.54 & 22.53 & 21.41 & 20.79 & 20.44 & 20.25 & 20.14 & 20.09 \\
0.0125 & 100 & 66.36 & 45.98 & 34.48 & 28.07 & 24.49 & 22.50 & 21.39 & 20.78 & 20.44 & 20.25 & 20.14 & 20.09 \\
0.015 & 100 & 65.13 & 45.15 & 34.01 & 27.80 & 24.34 & 22.42 & 21.35 & 20.75 & 20.42 & 20.24 & 20.14 & 20.09 \\
0.0175 & 100 & 62.98 & 43.76 & 33.22 & 27.36 & 24.10 & 22.28 & 21.27 & 20.71 & 20.40 & 20.22 & 20.13 & 20.08 \\
0.02 & 100 & 59.71 & 41.83 & 32.14 & 26.76 & 23.77 & 22.10 & 21.17 & 20.65 & 20.36 & 20.21 & 20.12 & 20.08 \\
\hline
\end{tabular}

\section{The solution of fluent}

The final temperature contour is shown in Figure 5.

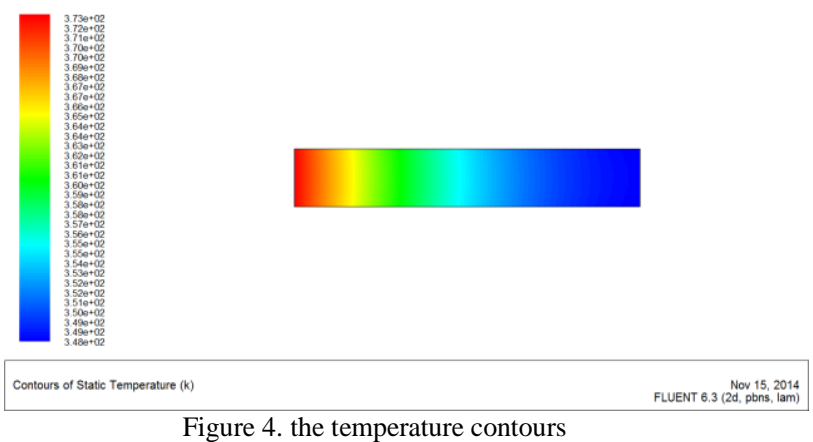

\section{RESULTS AND ERROR ANALYSIS}

Figure 5 shows the temperature results of each node which compares theoretical solution with the numerical solution. The results are in good agreement, which illustrate both theoretical solution and numerical solution can be used to solve this problem. 


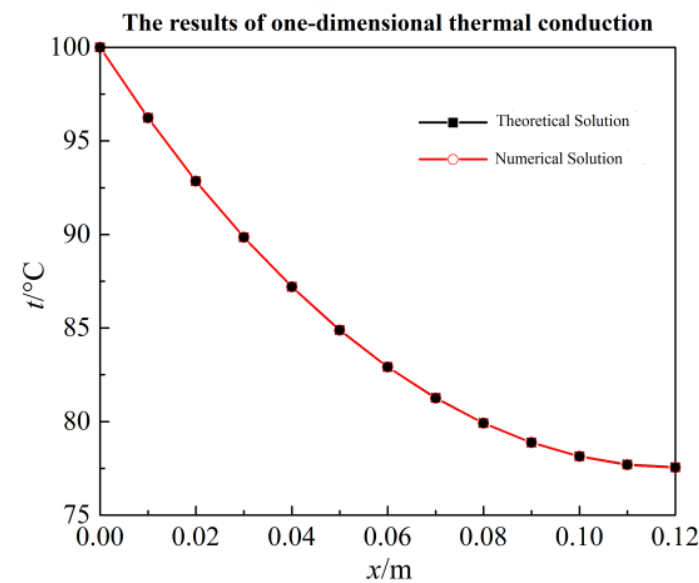

Figure 5. the temperature distribution curve of different solution methods
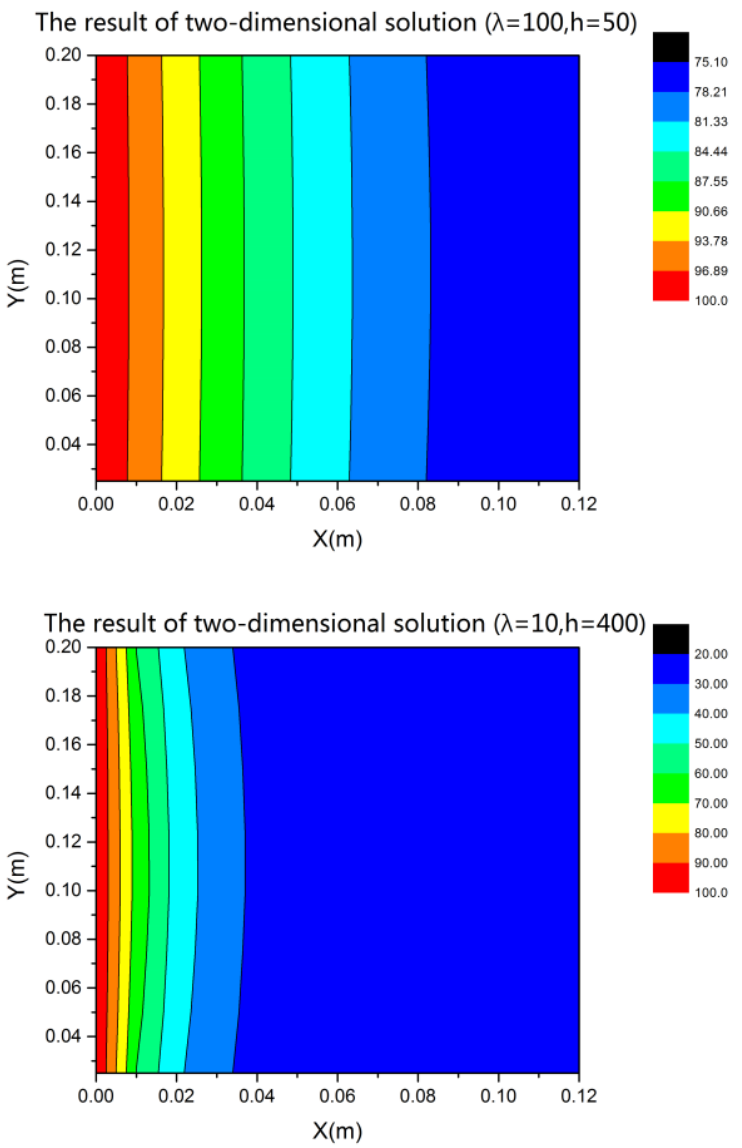

Figure 6. the two-dimensional temperature distribution field under different conditions

\section{CONCLUSIONS}

We can draw the conclusion from figure 6 , there are differences between the two-dimensional temperature distributions under different conditions. The causes are being analyzed as follows:

- The difference between minimum temperatures: In the first case, the minimum temperature is 75 degrees, the second case is 20 degrees, the surface heat transfer coefficient contributes a lot to the heat dissipation, which explains this differences.

- The difference between temperature distributions in the $\mathrm{x}$ direction: in the contrary near heat sources, the temperature gradient in the second case is significantly greater than the first case. The case which contains a lager thermal conductivity have a uniform temperature distribution and a lower temperature gradient, the smaller otherwise.

- The difference between temperature distributions in the y direction: The temperatures are almost the same at the center of the first case and the upper and lower boundaries. The temperatures are different at the center of the second case and the upper and lower boundaries. The relative size of conduction thermal resistance and the convection thermal resistance affects the temperature distribution in the y direction, when the convection thermal resistance is much less than the convective heat transfer resistance, the temperature distribution is uniform in the $\mathrm{y}$ direction, when the conduction thermal resistance is greater than convection thermal resistance, the situation is opposite.

$\bullet$

\section{References}

[1] Fabbri G. A genetic algorithm for fin profile optimization[J]. International Journal of Heat and Mass Transfer, 1997, 40(9): 2165-2172.

[2] Kraus A D, Aziz A, Welty J. Extended surface heat transfer[M]. John Wiley \& Sons, 2002.

[3] Yu L T. Application of Taylor transformation to optimize rectangular fins with variable thermal parameters[J]. Applied Mathematical Modelling, 1998, 22(1): 11-21.

[4] Mokheimer E M A. Performance of annular fins with different profiles subject to variable heat transfer coefficient[J]. International Journal of Heat and Mass Transfer, 2002, 45(17): 3631-3642.

[5] Sharqawy M H, Zubair S M. Efficiency and optimization of straight fins with combined heat and mass transfer-an analytical solution[J]. Applied Thermal Engineering, 2008, 28(17): 2279-2288. 